\title{
Clinical profile of amoebic liver abscess patients admitted in a tertiary care hospital

\author{
B Paik ${ }^{1}$, SK Sarkar ${ }^{2}$, PK Chowdhury ${ }^{3}$, S Ahmed ${ }^{4}$
}

\begin{abstract}
Amoebic liver abscess is an important cause of space occupying lesions of the liver, especially in tropical and sub tropical regions. It is the most frequent complication of invasive amebiasis. It may be found in all age groups but relatively rare in children. The signs and symptoms vary according to the severity of illness. The present study was done to evaluate the clinical profile of patients with amoebic liver abscess for age, gender, clinical features, site of abscess, number of abscess, treatment modality by intravenous metronidazole along with percutaneous needle aspiration and prognosis. A prospective study of 86 admitted patients of amoebic liver abscess were included in this study which was carried out in the medicine department of Khulna Medical College Hospital over a period of 2 years from July 2010 to June 2012. Mean age of patients was 45 years. Male female ratio was 7:1. The mean duration of fever was 17.9 days and the mean duration of pain was 14.1 days. The duration of fever for more than 2 weeks was seen in 38 cases. Regarding clinical features, the major symptoms of fever, pain abdomen and dysentery were seen in 81,78 and 10 cases respectively. The major signs as determined by clinical and radio imaging studies were hepatomegaly in 76, right lobe abscess in 60 , left lobe abscess in 12, multiple abscesses in both lobes in 18, ascites in 5 and right sided pleural effusion in 12 cases respectively. All the cases underwent percutaneous needle aspiration. 5 cases died out of 86 patients.
\end{abstract}

\section{Introduction}

Amoebiasis occurs in $10 \%$ of the world's population $1,2,4,5$ and is most common in tropical and sub tropical regions and in Bangladesh amoebic liver abscess accounts for 3-9\% of all cases of amoebiasis. ${ }^{3,4,20}$ Entamoeba histolytica causes dysentery, rectocolitis, and amoebic liver abscess. The clinical spectrum of intestinal disease is quite variable. Amoebic rectocolitis usually presents with non bloody diarrhea, milder abdominal discomfort, colicky pain and tenesmus but usually do not have fever or other systemic manifestations.2,4,5,7-9 Pathologically this protozoon causes inflammatory and ulcerative lesions of varying severity commonly in the cecum, sigmoid colon and rectum.7-10 Infection by Entamoeba histolytica is ubiquitous, but the highest endemic incidence is usually found in poor communities with non hygienic sanitation and people with a lower socio economic status. $3.4,7,20$

The occurrence of an amoebic liver abscess in a person who has not traveled to or resided in a non endemic area should raise the suspicion of underlying immunosupression, particularly AIDS.
Bang Med J Khulna 2015; 48 : 20-23

Other persons at increased risk include patients in residential institutions and men who have sex with men. Host factors that contribute to the severity of disease include young age, glucocorticoid use, and malignancy. $34,11,20$

During its life cycle, Entamoeba histolytica exists as trophozoite or cyst forms. After infection, amoebic cysts pass through the gastrointestinal tract and become trophozoites in the colon, where they invade the mucosa and produce typical "flask shaped" ulcers. The organism is carried by the portal vein circulation to the liver, where an abscess may develop. Occasionally, organisms travel beyond the liver and can produce abscess in the lung or brain. Rupture of an amebic liver abscess into the pleural, pericardial and peritoneal spaces can also occur.7,10

Amoebic liver abscess is 10 times more common in men than women and is rare in children. $2,5,7$ Symptoms are present on an average for 2 weeks by the time a diagnosis is made. A latency period between intestinal and subsequent liver infection of up to many years is possible, and less then $10 \%$ of patients report an antecedent history of bloody

1. Bishnupada Paik MD, Associate Professor, Dept. of Gastroenterology, Khulna Medical College, Khulna.

2. Swapan Kumar Sarkar MD, Associate Professor, Dept. of Gastroenterology, SBMCH, Barisal

3. Poritosh Kumar Chowdhury DTCD, Associate Professor, Dept. of Respiratory Medicine, Khulna Medical College.

4. Saad Ahmed M.Phil, Associate Professor, Dept. of Radiology \& Imaging, Kushtia Medical College, Kushtia. 
diarrhea with amoebic dysentery.3,7-9

Patients with amoebic liver abscess manifest early with abdominal pain well localised to the right hypochondrium. Fever is almost invariable but may be intermittent and sometimes presents as fever of unknown origin. Malaise, myalgia and arthralgia are common. Jaundice is uncommon and signifies a poor prognosis.2,5,10,11 Pulmonary symptoms and signs may be present but pericarditis and peritonitis are rare. $2-6,6-9$ The presentations of an amebic liver abscess is usually acute rather than a pyogenic liver abscess which usually presents in a subacute form. Pyogenic liver abscess is more common in the elderly people, more often multiple, usually polymicrobial and occurs in the setting of septicemia, abdominal surgery, peritonitis, diabetes mellitus and underlying immunosupression. 3,4,7,9,20

The diagnosis of ameobic liver abscess is based on clinical suspicion, laboratory features, serologic testing and hepatic imaging study. The protozoon is isolated from stool in only $50 \%$ of cases. ${ }^{2,5,7}$ Hepatic imaging studies cannot distinguish a pyogenic from an amebic liver abscess. 15 Amebic liver abscess is commonly localised to the right lobe, usually single and close to the diaphragm.2 4,7,9,15 Available serologic tests include an ELISA, indirect hemaglutination, counter immunoelectrophoresis and immunofluorescent antibody test.1315 Serologic test results must be interpreted in the clinical context, because serum antibody levels may remain elevated for years after recovery or cure. The sensitivity of these tests is approximately $95 \%$ and the specificity is over $95 \% .2,5,7$

Aspiration of an amebic liver abscess should be performed if the diagnosis remains uncertain or whether the response to treatment is slow.16-20 The presence of a reddish brown pasty aspirate ("anchovy paste" or "chocolate sauce") is typical; trophozoites rarely are indentified.7 Aspiration also may be considered in cases where an abscess in the left lobe is close to the pericardium or signs of impending rupture either to pleural space or peritoneum.2,9,16 Detection of Entameba histolytica DNA in saliva and urine could be used as a diagnostic tool for amebic liver abscess, but due to local nonavailability and cost we could not utilize DNA study by Real Time PCR assay in our study. 13

\section{Materials and methods}

This was a prospective study of 86 patients admitted in Khulna Medical College Hospital and diagnosed clinically by history and clinical features and then confirmed as amoebic liver abscess depending on laboratory studies including serology and ultrasonography and then treated by intravenous metronidazole along with percutanous aspiration.
Inclusion criteria were:

1. Abscess cavity size of $>5 \mathrm{~cm}$ on ultrasound examination

2. Left lobe abscess

Exclusion criteria were:

1. The presence of micro organisms other than Entameba histolytica on gram stain of pus or on culture

2. IgM-ELISA serology negative for Entamoeba histolytica

All data regarding the history and clinical examination with special attention to the liver size was recorded. Laboratory investigations recorded were complete blood count, liver function tests e.g. serum bilirubin, serum alanine aminotransferase, serum alkaline phosphatase. Data about blood cultures drawn for bacteria and pus drawn during percutaneous aspiration were noted. All patients were given therapy for amebic liver abscess in the form of a combination of intravenous metronidazole $800 \mathrm{mg} 8$ hourly for 10 days along with percutaneous aspiration.

For analysis of data, the statistical software SPSS version was used. A multivariate regression analysis was used to evaluate the relationship of rupture of liver abscess and mortality with various clinical features, ultrasonographic findings and treatment modalities. The Ethics committee of the Institute gave approval for this study. Informed written consent was taken from the patients or guardians of the study subjects.

\section{Results}

In a total of 86 patients, the male female ratio was $7: 1$. The mean (SD) age of patients was 12.38 with the range from 16 to 68 years. The most affected age groups were those between 31 to 40 years, followed by 41-50 years (Table 1).

Table I

Age group distribution of the patient.

\begin{tabular}{lcc}
\hline Age in years & No. of cases & $\%$ \\
\hline $11-20$ & 2 & 2.32 \\
$21-30$ & 8 & 9.30 \\
$31-40$ & 24 & 27.90 \\
$41-50$ & 22 & 25.58 \\
$51-60$ & 20 & 23.25 \\
$61-70$ & 10 & 11.62 \\
$\mathrm{SD} \pm 12.38$ & 86 & 100 \\
\hline
\end{tabular}

The mean duration of fever was 17.9 days (range $=$ 5-100 days) and the mean duration of pain was 
$14.1 \pm 1.7$ days. Symptoms duration of more than 2 weeks was seen in $52 \%$ of cases.

Table II

Major symptoms of the patients

\begin{tabular}{lcc}
\hline Symptoms & No. of cases & $\%$ \\
\hline Fever & 81 & $94 \%$ \\
Pain abdomen & 78 & $90 \%$ \\
Dysentery & 10 & $10.5 \%$ \\
\hline
\end{tabular}

Regarding clinical features of all patients, fever, pain abdomen and dysentery were seen in 94\%, 90\% and 10.5\% respectively, (Table II).

\section{Table III}

Internal features as determined by clinical and Radio imaging studies.

\begin{tabular}{lcc}
\hline Features & No. of patients & $\%$ \\
\hline Hepatomegaly & 76 & 88 \\
Abscess (Rt. lobe) & 60 & 65 \\
Abscess (Lt. Lobe) & 12 & 14 \\
Multiple abscesses (Both lobes) & 18 & 22 \\
Ascites & 5 & 5.70 \\
Pleural effusion (Rt) & 12 & 14 \\
\hline
\end{tabular}

Out of 86 patients, the liver could not be palpated below the right costal margin in 10 cases (11.6\%). A right sided pleural effusion was present in 12 cases (14\%), anemia in 53 cases $(62 \%)$ and leukocytosis in 59 cases (68\%). The serum hepatic transaminase levels were raised to more than 3 times normal in 30 cases (35\%). On ultrasound examination, a right lobe abscess was seen in 76 cases (65\%), left lobe abscess in 12 cases (14\%) and multiple abscesses in both lobes in 18 cases (22\%) (Table III).

4 patients had rupture of the abscess; right pleural rupture in I and peritoneal rupture in 3. The mean (SD) hospital stay of patients was 13.4 days (range $=2-35$ days). Although patients with ruptured amebic liver abscess had longer in hospital stay than those managed conservatively, yet this was not statistically significant. Out of 86 patients, 5 cases (5.8\%) died (male 4, female 1), (Fig 1).

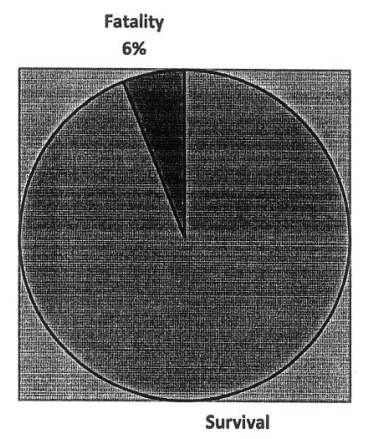

Fig 1. Pie-chart of survival and mortality

\section{Discussion}

The WHO estimates that Entameba histolytica causes 50 million cases and 100,000 deaths annually, making this disease the second leading cause of death from protozoal diseases.1-3,5-6 Although infection with Entameba histolytica occurs world wide, yet, liver abscess is the most common extra intestinal complication in 3-9\% of patients. 2.5.7,9,19-20 Diagnosis of amebic liver abscess is straightforward on the basis of epidemiological, clinical, serological and ultrasonographic findings. Amebic liver abscess arises from the hematogenous spread of the trophozoites of Entameba histolytica from the intestinal mucosa to the liver through the portal vein.

Male female ratio in our study is $7: 1$ which is similar to one study done in our country. 4 The disease in endemic areas, presented usually with fever, right hypochondriac pain and hepatic tenderness. ${ }^{4-7,9-10}$ Compared to pyogenic liver abscess, patients with amebic abscess are often younger, more acutely ill with fever and right hypochondriac pain, and are usually from high prevalence areas. The mean age of our patients with amebic liver abscess was 45 years and was comparable to other studies. $2-4,10,18,20$ In one study amebic liver abscess patients presented with fever (98.89\%), right hypochondriac pain (76.67\%), abdominal tenderness $(97.78 \%)$ and dysentery (7.78\%).3 But our study shows dysentery in (10\%) of cases. The frequency of fever and pain abdomen is $67-87 \%$ and $62-94 \%$ of patients with amebic liver abscess respectively in different series. In our study, these two symptoms of fever and pain abdomen occurred in 94\% and 90\% respectively. From India, Sharma et al in a study of 70 cases of amebic liver abscess found hepatomegaly in $84 \%$, pleural effusion in $10 \%$ and ascites in $4 \%$ cases respectively. ${ }^{17}$ Hepatomegaly $88 \%$ was a predominant feature of amebic liver abscess in our study as well. These findings correlate with other studies which show that clinically hepatomegaly, right hypochondriac pain and fever are major features of amebic liver abscess. ${ }^{4}$ We found pleural effusion in $14 \%$ and ascites in $5.7 \%$ cases respectively. In our study no cerebral manifestations was seen as amebic brain abscess is extremely rare. ${ }^{12}$

Jaundice occurred in $12.7 \%$ cases in our study. The pathophysiology of jaundice remains controversial and various explanations of jaundice have been proposed; such as pressure of abscess cavity on hepatic ducts and cholestasis.11 Duration of symptoms longer than 2 weeks in seen was $14-41 \%$ in different series. In a study of amebic liver abscess of 131 patients, the duration of symptoms less than 2 weeks was seen in $83.9 \%$ of cases.7,9

In this study, $84 \%$ of patients presented within 2 weeks and mild elevations of serum transaminase was seen in $19.8 \%$ cases. Duration of symptoms less than 2 weeks was evident in $48 \%$ cases and raised liver enzymes more than three times normal 
in $35 \%$ cases. Abdominal ultrasound is the gold standard for diagnosing liver abscess.15 Sonographically, in amebic liver abscess, 4-42\% cases have multiple abscesses, $20-35 \%$ have an abscess in the left lobe and the remaining 49-80\% have solitary abscess in the right lobe. 3,5,6,20 Our study showed multiple abscesses in $22 \%$ cases, a solitary left abscesses in $13 \%$ cases and a single right lobe abscesses in 65\% cases.

In one study of 90 cases rupture occurred in $4.44 \%$ cases. $^{3}$ In our study peritoneal rupture occurred in 3 cases and there was one case with right pleural rupture. ${ }^{18}$ None of our patients underwent surgical exploration. Although, the hospital stay of patients of ruptured amebic liver abscess was more than those managed conservatively, yet, it did not reach statistical significance. This may be due to small size of the group of patients managed conservatively.

In one study mortality rate in amebic liver abscess is seen in $3.33 \% .3$ The overall mortality rate in amebic liver abscess seen in various series ranges from 2-15\% $2,3,7-10,20$ but in our study the mortality rate was $5.8 \%$.

\section{Conclusion}

Now a days though amebic liver is less common but clinical suspicion in the endemic setting is highly valuable for early diagnosis and conservative management with metronidazole and percutaneous needle aspiration is both effective and approved as treatment modalities. It is expected that the findings of this present study would help to increase awareness among clinicians in regard to clinical features, early diagnosis, treatment modality and finally clinical outcome of amoebic liver abscess.

\section{Acknowledgement}

We thank all of our colleagues of Radiology department who assisted during our ultrasonography guided aspiration procedures. We are thankful to Dr. SM Khaliduzzaman, Lecturer, Dept. of Anatomy, KhuIna Medical College for statistical assistance. We are also thankful to Mr. Khairul Islam, Stenotypist, KMC for typing and Mr. S M Enamul Kabir, Librarian, $\mathrm{KMC}$ for kind co operation.

\section{References}

1. Ackers J, Clark CG, Diamond LS, et al: Amoebiasis. Bull World Health Org. 1997; 72: 97-100.

2. Haque R, Huston CD, Hughes M, et al: Amebiasis. N Eng J Med 2003; 348: 1565.

3. Alam F, Salam MA, Hassan P., Mahmood I, Kabir M, Haque $\mathrm{R}$ et al. Amebic liver abscess in northern region of Bangladesh: Sociodemographic determinants and clinical outcomes. BMC Research Notes 2014,7: 625.
4. Siddiqui MNA, Ahad MA, Ekram ARMS, Islam QT, Hoque MA, QAAI Masum et al. Clinico Pathological Profile of Liver Abscess in a Teaching Hospital. TAJ 2008; 21: 44-49.

5. Stanley SL Jr: Amoebiasis. Lancet 2003; 361:1015.

6. Li E, Stanley SL. Amebiasis. Gastroenterol Clin North Am 1996; 25: 471-492.

7. Raymond T. Chan\& Lawrence S. Friedman: Amebic liver abscess: In Sleisenger and Fordtran's Gastrointestinal and Liver Disease ed. Mark Feldman, Lawrence S, Friedman, Lawrence J, Brandt 8th ed. Philadelphia, Saunders, 2006; 17491753.

8. Stainikowitz DK, Sanchez RC. Amoebic liver Abscess: In Schiff's Diseases of the Liver; 8th Ed. Lippincott Raven Publishers; 1999; 1527-1532.

9. Carlos A. Dias Granado's, Wayme A Duffus, Duffin S, Helmut Albrecht: Parasitic Diseases of the Liver: In Hepatology ed. David Zakim, Thomas D. Boyer: 4th Ed, Philadelphia, Saunders 2003; 1073-1082.

10. Secto RK, Rockey DC: Amebic liver abscess: epidemiology, clinical features, and outcome. West $\mathrm{J}$ Med 1999; 170: 104- 109.

11. Thorsen S, Ronne Rasmussen J, Petersen, et al. Extra intestinal amebiasis. Clinical presentation in a non endemic setting. Scan J Infect Dis 1993; 25: 747-750.

12. De Villers JP, Durra G: Amebic abscess of the brain. Clin Radiol 1998; 53: 307-309.

13. Haque R, Kabir M, Noor Z, Rahman SM, Mondal D, Alam F, Rahman I, Mahmood AA, Nooruddin Ahmed, Williams A, Petri, Jr et al. Diagnosis of Amebic Liver Abscess and Amebic Colitis by Detection of Entamoeba histolytica DNA in Blood, Urine and Saliva by a Real Time PCR Assay. J Clin Microbiol, 2010; 48: 2798-2801.

14. Shamsuzzaman SM, Haque R, Hasin Sk. et al: Evaluation of Indirect fluorescent antibody test and enzyme linked immunosorbent assay for diagnosis of hepatic amebiasis in Bangladesh. J Parasitol 2000; 86: 622-815.

15. Kimura K, Stoopen M, Reeder MM et al: Amebiasis: modern diagnostic imaging with clinical and pathological correlation. Semin Roentgenol 1997; 32: 250-275.

16. Rajak CL, Gupta S, Jain S, et al: Percutaneous treatment of liver abscess: needle aspiration versus catheter drainage AJR Am J Roetgenol 1998; 170: 1035-1039.

17. Sharma MP, Dasarthy S, Verma M, Saksena S, Shukla DK. Prognostic markers in amebic liver abscess: a prospective study. Am J Gastoenterol, 1996; 91: 2584-2588.

18. Petri WA, Singh V: Diagnosis and management of amebiasis: Clin Infect Dis 1999; 29: 1117-1125.

19. Sharma MP, Ahuja V: Management of amebic liver abscess. Arch Med Res, 2000; 31: 54-55.

20. Roy PK, Raihan ASMA, Saha Sk, et al. Liver abscess: Clinical Profile and Therapeutic response. Bangladesh, J. Medicine. 2000; 11: 28-30. 